\title{
Hierarchical Microstructure in Keratin Biofibers
}

\author{
A.L. Martínez-Hernández ${ }^{1,2}$, C. Velasco-Santos ${ }^{1,2}$, M. de Icaza ${ }^{1,2}$ and V.M. Castaño ${ }^{1,2}$. \\ ${ }^{1}$ Centro de Física Aplicada y Tecnología Avanzada, Universidad Nacional Autónoma de México, \\ A.P. 1-1010, Santiago de Querétaro, Querétaro 76000, MÉXICO; castano@,fata.unam.mx. \\ ${ }^{2}$ Universidad Autónoma de Querétaro, Centro Cerro de las Campanas, Santiago de Querétaro, \\ Querétaro 76010, MÉXICO.
}

Introduction.

It is possible to find in Nature an almost infinite source of high performance materials which remain to be seriously studied to establish them as basis for innovative technologies and useful raw materials. This is the case of keratin fiber from chicken feathers. Keratin, considered as the main structural component of these materials, contributes to a wide range of essential functions, including temperature control and physical and chemical protection. Structural studies of hair fibers, including wool, reveal highly organized subcomponents that could be find also in feather fibers starting from their complex branched structure of keratinaceous filaments that grow by a unique mechanism from cylindrical feather follicles. This branching structure, a distinctive morphological feature of feathers, has its origin in the biological evolution of feathers [1].

Microstructural study.

Barbs coming from the thick central barb and also nodes along each barb are shown in figure 1; it can be assumed that the nodes are similar to the cuticle scales in wool. The cuticle consists of layers of scales and its function is to protect the fiber's cortex. Nodes and barbs on the feather fiber are related to "memory" properties and improve structural strength. Keratin fibers have a high degree of flexibility, mechanical property that depends on its small diameter, high elastic modulus and shape, and that should be used to modify bulk elastic properties of new materials [2].

As shown in figures 1 and 2, feathers are endowed with a hierarchical structure starting at the level of the central barbs which grow directly from the quill. These central barbs are tiny "quills" which, in turn, also grow barbs. Notice in figure $2 \mathrm{~b}$ that central barbs are hollow and have an irregular shapes (non cylindrical). Surface characteristics of keratin fibers may be observed in figures $2 \mathrm{c}$ and $2 \mathrm{~d}$, where, at the same time, we can observe that these fibers are not hollow as tubes, but are rather filled. The cleft lines or striations along the fibers give rise to a certain surface roughness, which can contribute to interfacial strength that in addition to the high length to diameter ratio reached for the fiber showed in figure $2 \mathrm{e}$, can be useful for reinforcing composites (one of the possible applications for this fiber) [3].

Figure 3 shows the TEM micrographs in which the microfibrils are observed immersed in the amorphous matrix, identified by its dark color. The microfibrils are twisted forming an helix that is responsible of the fiber's high mechanical strength. In the figure $3 \mathrm{~b}$ is confirmed the presence of two different structures inside the biofibers: microfibrils and protofibrils. The former has a more order and crystalline structure than the matrix, being this amorphous and with a high content of cystine revealed by the reaction between the sulphur contained in the aminoacid and the fixation and 
contrast chemical agents. The protofibrils are inside the microfibrils and are also surrounded by the matrix as can be appreciated in the figure $3 \mathrm{~b}$.

\section{Conclusions.}

The microscopic study permit us observe and describe the feather fibers characteristics: hierarchical and highly ordered structure, starting from their internal parts in TEM. The fibers are by themselves complex natural composites with important and interesting characteristics, useful for different applications, that are being reported elsewhere.

\section{References.}

[1] X. Xu, Z. Zhou, R.O. Prum, Nature, 410, (2001), 200.

[2] K.K. Chawla, Composite Materials Science and Engineering, $2^{\text {nd }}$. Ed. Springer-Verlag, 1998.

[3] A.L. Martínez-Hernández, C. Velasco-Santos, M. de Icaza, V.M. Castaño, Environmental

Forensics, in press (2003).

[4] The aid of Mrs. J. Cañetas for taking the SEM and to Mrs M.L. Palma for taking the TEM is gratefully acknowledged.

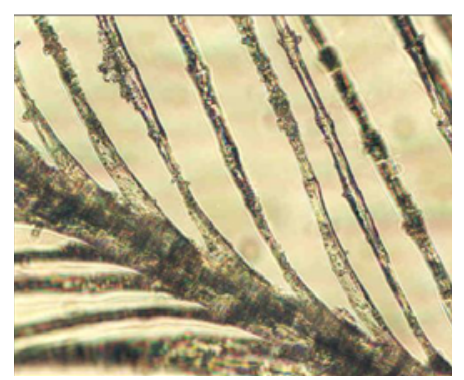

Fig. 1. Optical microscopy image of feather fiber.
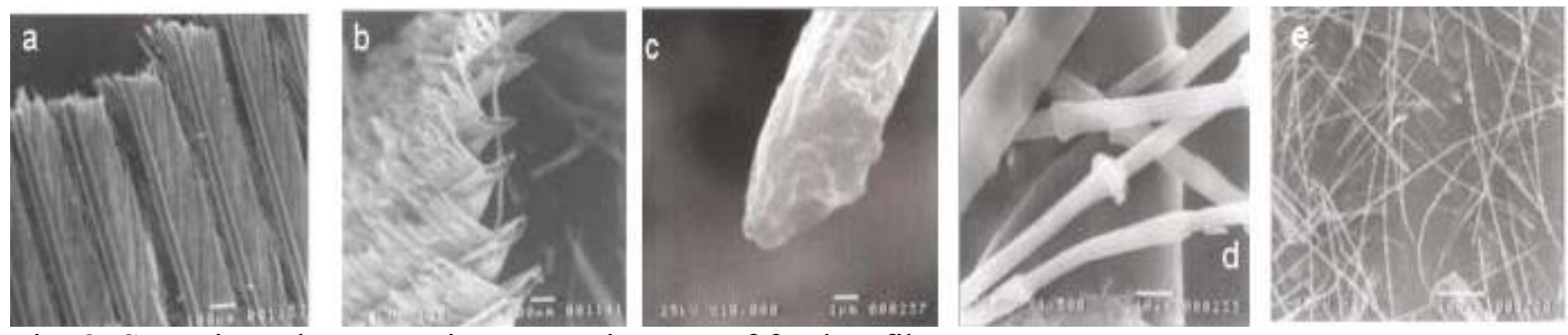

Fig. 2. Scanning Electron Microscopy images of feather fiber.
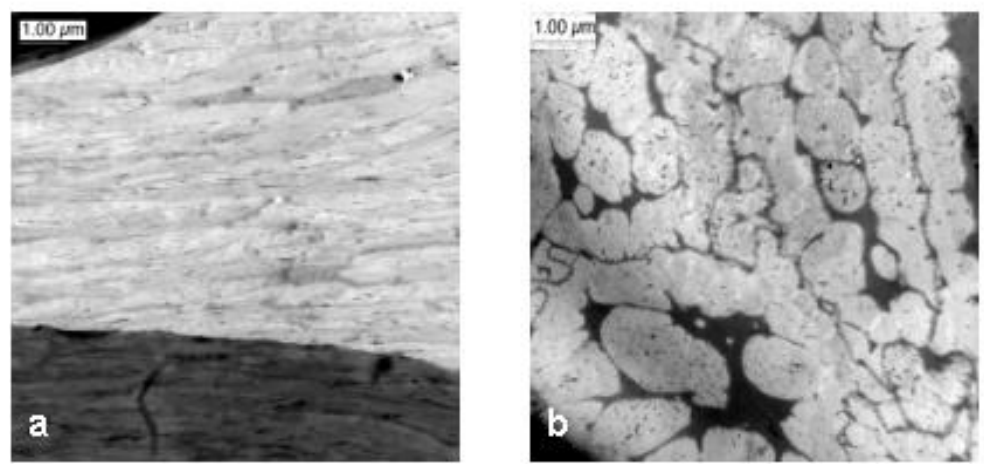

Fig. 3. Transmission Electron Microscopy images: a) longitudinal section, b) cross section of feather fiber. 